\title{
Magnetic resonance imaging identifies early effects of sunitinib treatment in human melanoma xenografts
}

\author{
Jon-Vidar Gaustad*, Viktoria Pozdniakova, Tord Hompland, Trude G Simonsen and Einar K Rofstad
}

\begin{abstract}
Background: Antiangiogenic treatment may change the tumor microenvironment and hence influence the effect of conventional therapies. The potential of diffusion weighted magnetic resonance imaging (DW-MRI) and dynamic contrast enhanced MRI (DCE-MRI) in assessing microenvironmental effects of sunitinib treatment was investigated in this preclinical study.
\end{abstract}

Methods: Sunitinib-treated and untreated A-07 tumors were subjected to DW-MRI and DCE-MRI, and parametric images of $A D C$ and $K^{\text {trans }}$ were produced. Microvascular density, hypoxic fraction, and necrotic fraction were assessed from immunohistochemical preparations, and tumor interstitial fluid pressure (IFP) was assessed with probe measurement.

Results: Sunitinib-treated tumors showed reduced microvascular density, increased hypoxic fraction, increased necrotic fraction, increased ADC, and reduced $K^{\text {trans }}$, but did not differ from untreated tumors in growth rate and IFP.

Conclusions: Sunitinib treatment affected the tumor microenvironment without affecting tumor size. DW-MRI and DCE-MRI were sensitive to the sunitinib-induced changes in the tumor microenvironment.

Keywords: Sunitinib, Antiangiogenic treatment, Hypoxia, DW-MRI, DCE-MRI

\section{Background}

Several antiangiogenic drugs are being investigated, including endogenous inhibitors of angiogenesis [1], monoclonal antibodies against pro-angiogenic factors or their receptors [2,3], and small molecule tyrosine kinase inhibitors which may target multiple pro-angiogenic receptors [4]. The antiangiogenic agents are generally not cytotoxic, and treatment-induced reductions in tumor size often appear late compared to vascular effects [5]. It is therefore recognized that functional parameters are more appropriate than tumor size for evaluating early effects of antiangiogenic treatment [6].

Antiangiogenic therapy may inhibit tumor growth significantly when used as a single treatment modality, but the therapeutic benefit may be even greater when used in combination with conventional treatment modalities

\footnotetext{
* Correspondence: Jon.Vidar.Gaustad@rr-research.no

Department of Radiation Biology, Group of Radiation Biology and Tumor Physiology, Institute for Cancer Research, Oslo University Hospital, Montebello, Oslo N-0310, Norway
}

such as radiation and chemotherapy [7]. Tumor response to radiation and chemotherapy can be significantly affected by the tumor microenvironment. Tumors with extensive hypoxia are more resistant to radiation and some forms of chemotherapy, and elevated interstitial fluid pressure (IFP) may reduce the uptake of chemotherapeutic drugs [8,9]. Antiangiogenic treatment has been reported to improve oxygenation and reduce IFP in some tumor models $[2,3]$ and to induce hypoxia in others $[10,11]$. The reasons for these different effects are not clear, but the effects have important implications for combination therapies. Careful monitoring of the tumor microenvironment during antiangiogenic treatment may help to optimize the timing of combination therapies.

Tumor response to antiangiogenic treatment has been evaluated with diffusion weighted magnetic resonance imaging (DW-MRI) and dynamic contrast-enhanced MRI (DCE-MRI) [6,12]. DW-MRI is sensitive to the Brownian motion of water molecules which is restricted by cell membranes and extracellular fibers in tissues [12]. 
The apparent diffusion coefficient (ADC) is often used to quantify DW-MRI data, and this parameter has been shown to reflect cell density and to be sensitive to necrotic tissue in untreated tumors [12,13]. Moreover, both reductions and increases in tumor ADC have been reported after antiangiogenic treatment $[14,15]$. In DCE-MRI, the uptake of a paramagnetic contrast agent is studied by imaging tumors before and multiple times within a few minutes after the injection of the contrast agent. The transfer rate constant, $K^{\text {trans }}$, can be estimated by using the generalized pharmacokinetic model of Tofts to analyze DCE-MRI series [16]. $K^{\text {trans }}$ generally reflects blood perfusion and the vessel permeability - vessel surface area product [17]. When using low molecular weight contrast agents like Gd-DTPA $(550 \mathrm{Da}), K^{\text {trans }}$ has been shown to reflect blood perfusion in untreated tumors with high vessel permeability [18]. Reductions in $K^{\text {trans }}$ or $K^{\text {trans }}$-related parameters have been reported in most studies evaluating tumor response to antiangiogenic agents with DCE-MRI [6]. A weakness in many of the studies evaluating tumor response to antiangiogenic treatment with DW-MRI and/or DCEMRI is that treatment-induced effects on the tumor microenvironment were not assessed with non-MR techniques. Consequently, it is not always clear how the changes in MR-derived parameters were related to the tumor microenvironment.

Sunitinib is a small molecule tyrosine kinase inhibitor which targets vascular endothelial growth factor receptors 1-3 (VEGFR-1, -2, and -3), platelet-derived growth factor receptors $\alpha-\beta$ (PDGFR- $\alpha$ and PDGFR- $\beta$ ), stem cell growth factor receptor (c-KIT), and fms-like tyrosine kinase receptor 3 (FLT 3) [19]. Sunitinib has been shown to prolong progression-free and overall survival in patients with imatinib-refractory gastrointestinal stromal tumor, metastatic renal cell carcinoma, and progressive, welldifferentiated pancreatic neuroendocrine tumor in clinical phase III trials, and has been approved by the US Food and Drug Administration for these indications [20-22]. In the current study we evaluated the effect of sunitinib treatment on the tumor microenvironment by using histological techniques to assess microvessels, tumor hypoxia, and tumor necrosis and probe measurement to assess tumor IFP. We also evaluated the effect of sunitinib treatment with DW-MRI and DCE-MRI. We report that sunitinib treatment increased ADC and reduced $K^{\text {trans }}$, reflecting sunitinib-induced tumor necrosis and sunitinib-induced reductions in tumor microvascular density and oxygenation.

\section{Methods}

Mice and tumors

Adult (8-12 weeks of age) female BALB/c-nu/nu mice, bred at our research institute, were used as host animals for xenografted tumors. Animal care and experimental procedures were approved by the Institutional Committee on Research Animal Care and were performed in accordance with the Interdisciplinary Principles and Guidelines for the Use of Animals in Research, Marketing, and Education (New York Academy of Sciences, New York, NY, USA). The experiments were performed with tumors of the amelanotic human melanoma A-07, established and characterized as described previously [23]. A-07 cells were obtained from our frozen stock and were cultured in RPMI-1640 medium (25 mM HEPES and L-glutamine) supplemented with $13 \%$ bovine calf serum, $250 \mathrm{mg} / \mathrm{l}$ penicillin, and $50 \mathrm{mg} / \mathrm{l}$ streptomycin. Approximately $3.5 \times 10^{5}$ cells in $10 \mu \mathrm{l}$ of Hanks' balanced salt solution (HBSS) were inoculated intradermally in the hind leg by using a $100-\mu l$ Hamilton syringe. Tumor volume $(V)$ was calculated as $V=(\pi / 6) \times a \times b^{2}$, where $a$ is the longer and $b$ is the shorter of two perpendicular diameters, measured with calipers.

\section{Sunitinib treatment}

Sunitinb L-malate (LC Laboratories, Woburn, MA, USA) was dissolved in hydrochloric acid (1.0 molar ratio of sunitinib). Polysorbate 80 (0.5\%; Sigma-Aldrich, Schnelldorf, Germany), polyethylene Glycol 300 (10\%; Sigma-Aldrich), sodium hydroxide (to adjust $\mathrm{pH}$ to 3.5 ), and sterile water were added to the solution. Mice were treated with $40 \mathrm{mg} / \mathrm{kg} /$ day sunitinib or vehicle for 4 days, by oral administration.

\section{Anesthesia}

MRI and IFP measurements were carried out with anesthetized mice. Fentanyl citrate (Janssen Pharmaceutica, Beerse, Belgium), fluanisone (Janssen Pharmaceutica), and midazolam (Hoffmann-La Roche, Basel, Switzerland) were administered intraperitoneally in doses of $0.63 \mathrm{mg} / \mathrm{kg}$, $20 \mathrm{mg} / \mathrm{kg}$, and $10 \mathrm{mg} / \mathrm{kg}$, respectively. The body core temperature of the mice was kept at $37-38^{\circ} \mathrm{C}$ during MRI and IFP measurements by using a thermostatically regulated heating pad.

\section{MRI}

MRI was performed by using a 1.5 -T whole-body clinical scanner (Signa; General Electric, Milwaukee, WI, USA) and a slotted tube resonator transceiver coil constructed for mice. The tumors were positioned in the isocenter of the magnet and were imaged axially in a single section through the tumor center.

DW-MRI was carried out by applying a diffusionweighted single-shot fast spin echo sequence with ETL = 84 and TR $=5002 \mathrm{~ms}$. The diffusion weighted images were recorded at a spatial resolution of $0.39 \times 0.39 \times 2.0 \mathrm{~mm}^{3}$ by using an image matrix of $256 \times 256$, a field of view of $10 \times 10 \mathrm{~cm}^{2}$, and 5-10 excitations. Diffusion sensitization 
gradients were applied in six non-collinear directions with the following $\mathrm{x}, \mathrm{y}$, and $\mathrm{z}$ physical gradient combinations:

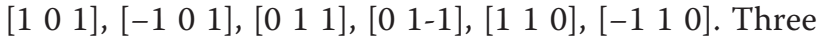
different diffusion-weightings with diffusion encoding constants of $b=200,400$, and $800 \mathrm{~s} / \mathrm{mm}^{2}$ and corresponding echo times of $\mathrm{TE}=85,95.5$, and 108.9 ms were used. An image without diffusion weighting $(b=0)$ was recorded for each TE value to compensate for the different TEs associated with the different $b$ values. The total scan time of our DW-MRI method was $\sim 10 \mathrm{~min}$. ADC maps were produced with in-house-made software developed in Matlab. Briefly, the directional diffusion images were averaged on a voxel-by-voxel basis to non-directional diffusion images. ADC values were calculated for each voxel by fitting signal intensities $(S)$ to the monoexponential model equation:

$$
\log \left(\frac{S(b, \mathrm{TE})}{S(b=0, \mathrm{TE})}\right)=-b \cdot A D C+c
$$

by using a linear least square fit algorithm. The signal decay of a large number of voxels was investigated to verify that the mono-exponential model gave good fits to the data. The fits generally had a correlation coefficient of $0.98-0.99$.

DCE-MRI was carried out as described earlier [24]. Briefly, Gd-DTPA (Schering, Berlin, Germany), diluted to a final concentration of $0.06 \mathrm{M}$, was administered in the tail vein of mice in a bolus dose of $5.0 \mathrm{ml} / \mathrm{kg}$ during a period of $5 \mathrm{~s}$. Two calibration tubes, one with $0.5 \mathrm{mM}$ Gd-DTPA in $0.9 \%$ saline and the other with $0.9 \%$ saline only, were placed adjacent to the mice in the coil. The tumors and the calibration tubes were imaged at a spatial resolution of $0.23 \times 0.23 \times 2.0 \mathrm{~mm}^{3}$ by using an image matrix of $256 \times 128$, a field of view of $6 \times 3 \mathrm{~cm}^{2}$, and one excitation. Two types of spoiled gradient recalled images were recorded: proton density images $(\mathrm{TR}=900 \mathrm{~ms}$, $\mathrm{TE}=3.2 \mathrm{~ms}$, and $\left.\alpha_{\mathrm{PD}}=20\right)$ and $T_{1}$-weighted images $\left(\mathrm{TR}=200 \mathrm{~ms}, \mathrm{TE}=3.2 \mathrm{~ms}\right.$, and $\left.\alpha_{\mathrm{T} 1}=80\right)$. The durations of the imaging sequences were 64 and $14 \mathrm{~s}$, respectively. Two proton density images and three $T_{1}$-weighted images were acquired before Gd-DTPA was administered. After the administration of Gd-DTPA, $T_{1}$-weighted images were recorded every $14 \mathrm{~s}$ for $15 \mathrm{~min}$. Gd-DTPA concentrations were calculated from signal intensities by using the method of Hittmair et al. [25]. The DCE-MRI series were analyzed on a voxel-by-voxel basis by using the arterial input function of Benjaminsen et al. [24] and the Tofts pharmacokinetic model [16] to produce parametric images of $K^{\text {trans }}$.

\section{IFP measurements}

IFP was measured by using a Millar SPC 320 catheter equipped with a $2 \mathrm{~F}$ Micro-Tip transducer with diameter
$0.66 \mathrm{~mm}$ (Millar Instruments, Houston, TX) [26]. The catheter was connected to a computer via a Millar TC-510 control unit and a model 13-66150-50 preamplifier (Gould Instruments, Cleveland, OH). IFP was measured in the center of the tumors by placing the catheter $5-10 \mathrm{~mm}$ from the tumor surface. Marks on the catheter assured correct positioning of the sensor, and a single measurement was carried out in each tumor. Only IFP measurements with stable readings for 3-5 minutes were accepted, and the measurements lasted for 10-20 minutes. Data acquisition was carried out by using LabVIEW software (National Instruments, Austin, TX).

\section{Hypoxia, necrosis, and microvessels}

CD31 was used as a marker for endothelial cells and pimonidazole [1-[(2-hydroxy-3-piperidinyl)-propyl]-2-nitroimidazole] was used as a hypoxia marker. Pimonidazole was dissolved in $0.9 \%$ sodium chloride and administered intraperitoneally at a dose of $30 \mathrm{mg} / \mathrm{kg}$. The tumors were resected and fixed in phosphate-buffered $4 \%$ paraformaldehyde approximately 4 hours after the pimonidazole administration. Immunohistochemistry was done by using a peroxidase-based indirect staining method [27]. An anti-pimonidazole rabbit polyclonal antibody (gift from Prof. J.A. Raleigh, Department of Radiation Oncology, University of North Carolina School of Medicine, Chapel Hill, NC) or an anti-CD31 rabbit polyclonal antibody (Abcam, Cambridge, United Kingdom) was used as primary antibody. Diaminobenzidine was used as chromogen, and hematoxylin was used for counterstaining. Hypoxic fraction was defined as the area fraction showing positive pimonidazole staining (hypoxic fraction $=$ pimonidazole positive area/viable tissue area.100\%) and necrotic fraction was defined as the area fraction showing necrotic tissue (necrotic fraction $=$ necrotic tissue area/total area $100 \%$ ). The area fraction showing positive pimonidazole staining and the area fraction showing necrotic tissue were determined by image analysis. Microvascular density was defined as the number of microvessel profiles per $\mathrm{mm}^{2}$ of viable tumor tissue (microvascular density = number of microvessel profiles/viable tissue area). The number of microvessel profiles was scored manually in immunohisochemical preparations stained with anti-CD31 antibody.

\section{Statistical analysis}

Statistical comparisons of data were carried out by the Student's $t$ test when the data complied with the conditions of normality and equal variance. Under other conditions, comparisons were done by nonparametric analysis using the Mann-Whitney rank sum test. Probability values of $P<0.05$, determined from two-sided tests, were considered significant. The statistical analysis was performed by using the SigmaStat statistical software (SPSS Science, Chicago, IL, USA). 


\section{Results}

A-07 tumors were divided into groups with matched tumor sizes to receive sunitinib treatment or no treatment (vehicle). Tumors in both groups grew during the 4-day treatment period (Figure 1). After the treatment, sunitinib-treated tumors did not differ from untreated tumors in size (Figure $1 ; P>0.05$ ), indicating that this short-term treatment did not affect tumor growth.

Sunitinib treatment affected tumor physiology. This is illustrated in Figure 2 which shows representative immunohistochemical preparations stained for microvessels (Figure 2A) and hypoxia (Figure 2B), and graphs illustrating the quantification of microvascular density, hypoxic fraction, necrotic fraction, and tumor IFP in untreated and sunitinib-treated tumors (Figure 2C-F). Sunitinib-treated tumors showed lower microvascular densities (Figure $2 \mathrm{C} ; P<0.0001$ ), higher hypoxic fractions (Figure 2D; $P=0.045$ ), and higher necrotic fractions (Figure 2E; $P=0.0015$ ) than untreated tumors. Sunitinibtreated tumors did not differ from untreated tumors in IFP (Figure 2F; $P>0.05$ ).

To investigate whether MRI could detect sunitinbinduced changes in tumor physiology, untreated and sunitinib-treated tumors were subjected to DW-MRI and DCE-MRI. ADC images and ADC frequency distributions were produced from DW-MRI data, and $K^{\text {trans }}$ images and $K^{\text {trans }}$ frequency distributions were produced from DCE-MRI series. Figure 3 shows the ADC image, the corresponding ADC frequency distribution, the $K^{\text {trans }}$ image, and the corresponding $K^{\text {trans }}$ frequency distribution of a representative untreated tumor (Figure 3A) and a representative sunitinib-treated tumor (Figure 3B). Figure 4 shows average ADC and average $K^{\text {trans }}$ of 15 untreated and 14 sunitinb-treated tumors, demonstrating that sunitinib-treated tumors showed significantly higher ADC values (Figure 4A; $P<0.0001$ ) and significantly lower

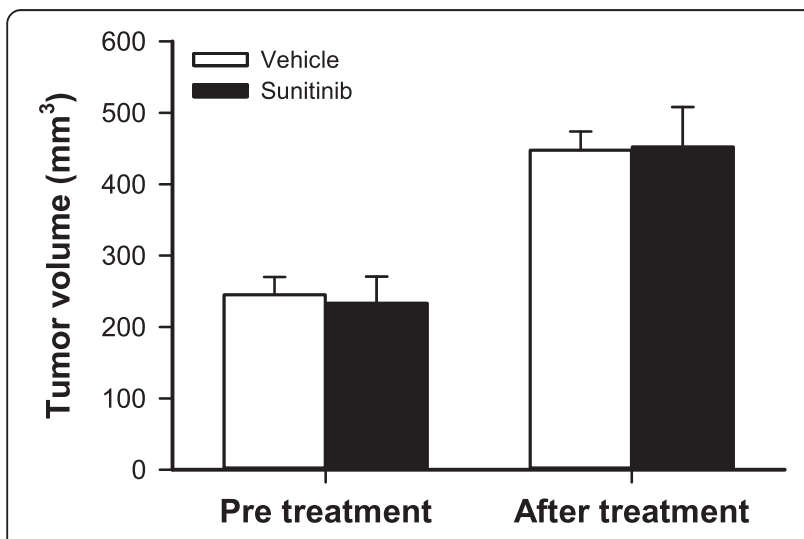

Figure 1 Sunitinib treatment did not affect tumor growth. Tumor size before and after 4 days of treatment in mice given vehicle (white colomns) or sunitinib (black columns). Columns, means of 14-15 A-07 tumors, bars SEM.
$K^{\text {trans }}$ values (Figure 4B; $P=0.0037$ ) than untreated tumors.

\section{Discussion}

Sunitinib treatment did not reduce the growth of A-07 tumors, but despite this sunitinib-treated tumors showed altered vasculature and microenvironment and, interestingly, altered ADC and $K^{\text {trans }}$ values. These observations illustrate that sunitinib treatment affected tumor physiology without affecting tumor size, and that DW-MRI and DCE-MRI were sensitive to these early effects. The observation that this short sunitinib treatment did not affect tumor growth is in line with our previous experience with tumors of the same melanoma line growing in dorsal window chambers [11]. In that study, we observed that 4-days with sunitinib treatment did not affect tumor growth, whereas tumor growth was reduced when the treatment was continued for 8 days.

Treatment-induced reductions in tumor size generally occur late after antiangiogenic treatment [5]. If nonresponding patients could be identified shortly after treatment initiation, any ineffective treatment could be stopped to avoid toxicity, and other treatments could be considered. In the current study, a short treatment period was chosen deliberately to investigate whether DW-MRI and DCE-MRI can detect treatment-induced effects occurring before reductions in tumor size. Our study suggests that these MR techniques may be used to identify patients that respond to antiangiogenic treatment before treatment-induced reductions in tumor size can be detected.

Sunitinib-treated tumors showed reduced $K^{\text {trans }}$ and increased ADC values. The reduction in $K^{\text {trans }}$ could be attributed to several vascular effects, but sunitinib-induced reduction in microvascular density was probably the dominating effect. We have previously shown that $K^{\text {trans }}$ reflects vessel density in untreated A-07 tumors $[24,28]$, and in the current study sunitinib-treated tumors showed significantly lower microvascular density than untreated tumors. Sunitinib-induced inhibition of VEGFR2 may also have reduced vessel permeability, because VEGF-A signaling is known to increase vessel permeability [29]. The reduction in $K^{\text {trans }}$ may thus also be influenced by reduced vessel permeability. The increase in ADC was probably a result of sunitinib-induced necrosis. Sunitinib-treated tumors showed massive necrosis whereas untreated tumors did not show necrotic regions. Elevated $\mathrm{ADC}$ values have been found in necrotic tissue in untreated tumors [12,13], and increases in ADC reflecting treatment-induced necrosis have been reported after chemotherapy, radiation therapy, and treatment with vascular disrupting agents [6].

In the current study, DW-MRI was performed by choosing $b$ values of $200-800 \mathrm{~s} / \mathrm{mm}^{2}$ to avoid confounding 


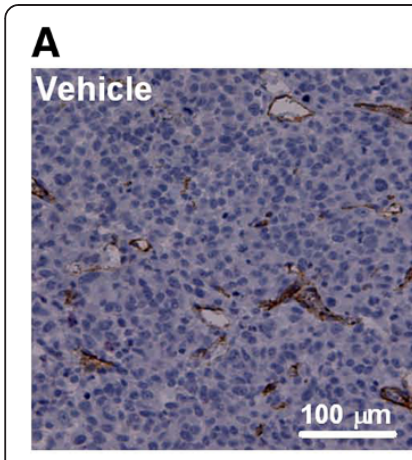

\section{C}

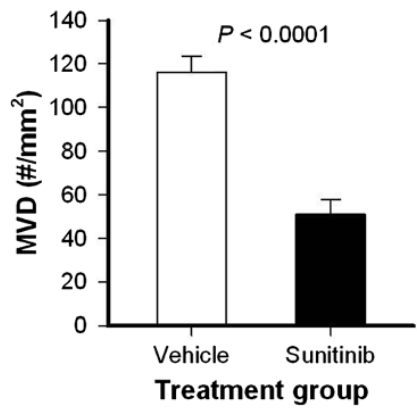

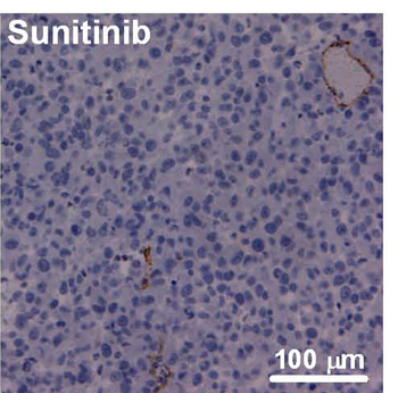

D

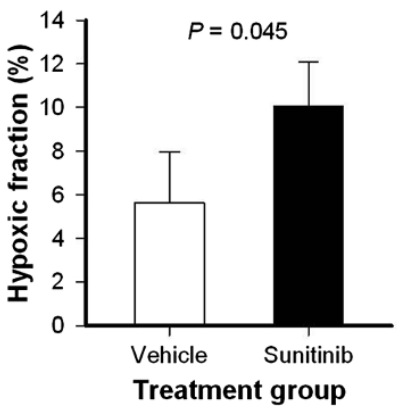

B

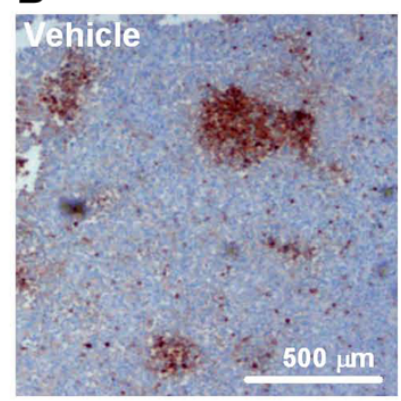

E

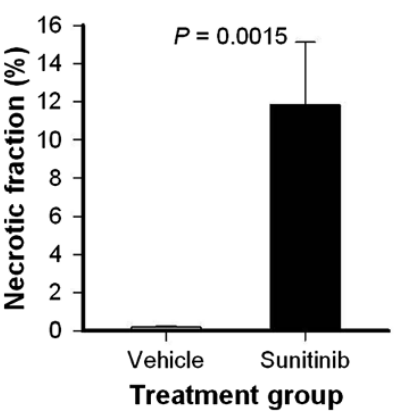

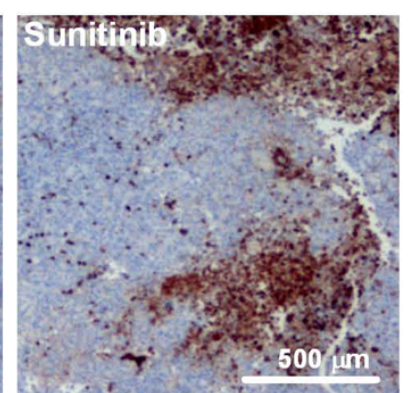

$\mathbf{F}$

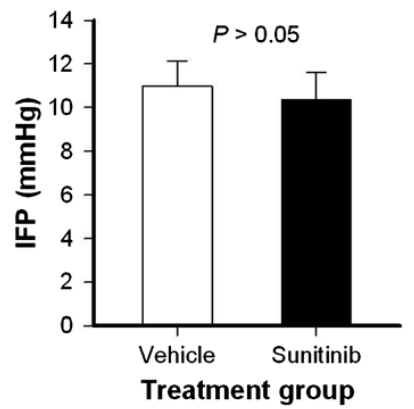

Figure 2 Sunitinib treatment affected tumor physiology. A-B, representative immunohistochemical preparations stained with anti-CD31 antibody to visualize microvessels (A) or anti-pimonidazole antibody to visualize hypoxic regions (B). The images show an untreated A-07 tumor (vehicle; left) and a sunitinib-treated A-07 tumor (sunitinib; right). C-F, microvascular density (MVD), hypoxic fraction, necrotic fraction, and IFP in untreated and sunitinib-treated A-07 tumors. Columns, means of 11-15 tumors; bars, SEM.

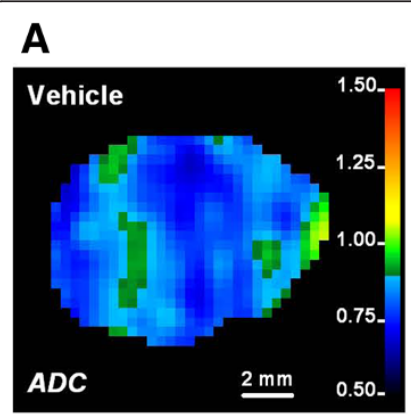

B

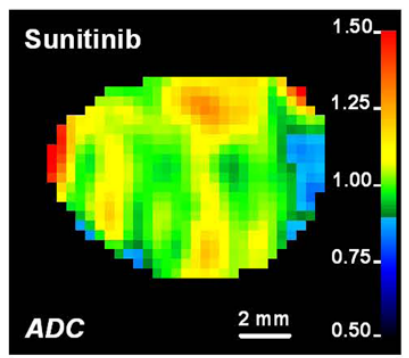

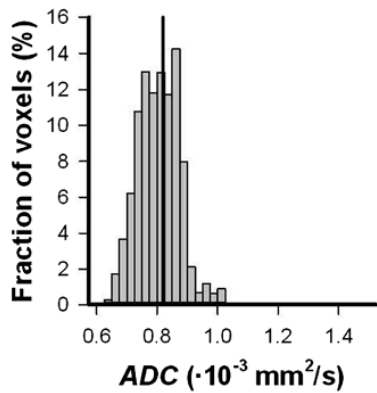

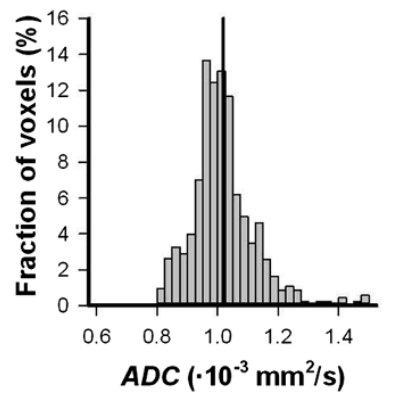

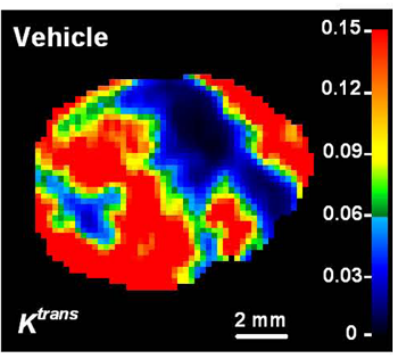
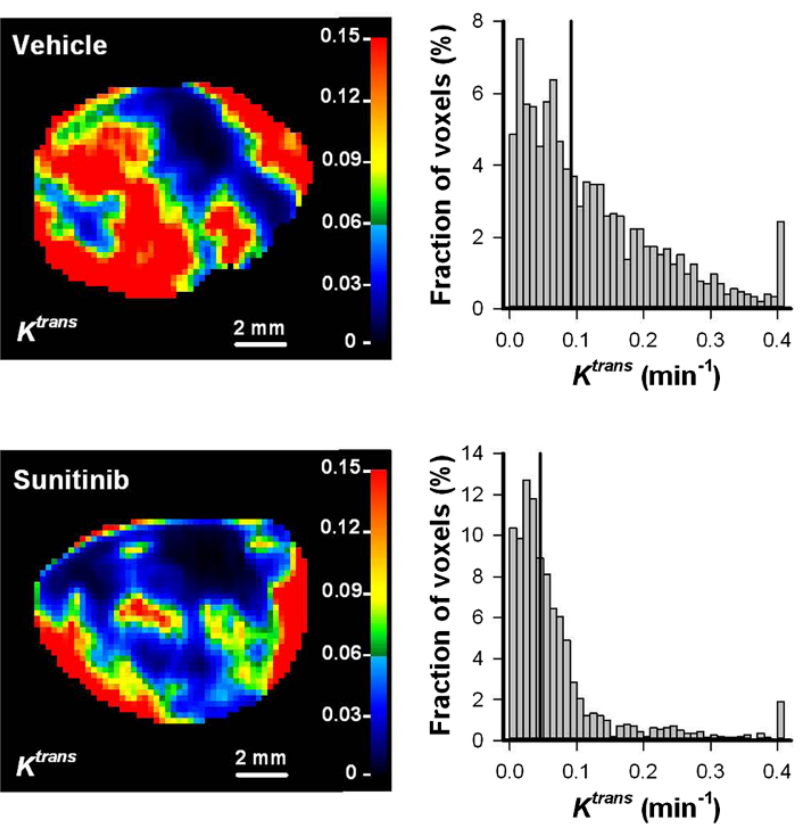

Figure 3 ADC and $K^{\text {trans }}$ images. ADC image, the corresponding ADC frequency distribution, $K^{\text {trans }}$ image, and the corresponding $K^{\text {trans }}$ frequency distribution of a representative untreated A-07 tumor (A) and a representative sunitinib-treated A-07 tumor (B). Color bars show ADC scale in $10^{-3} \mathrm{~mm}^{2} / \mathrm{s}$ or $K^{\text {trans }}$ scale in $\mathrm{min}^{-1}$. Vertical line in the frequency distributions shows median ADC or median $K^{\text {trans }}$. 

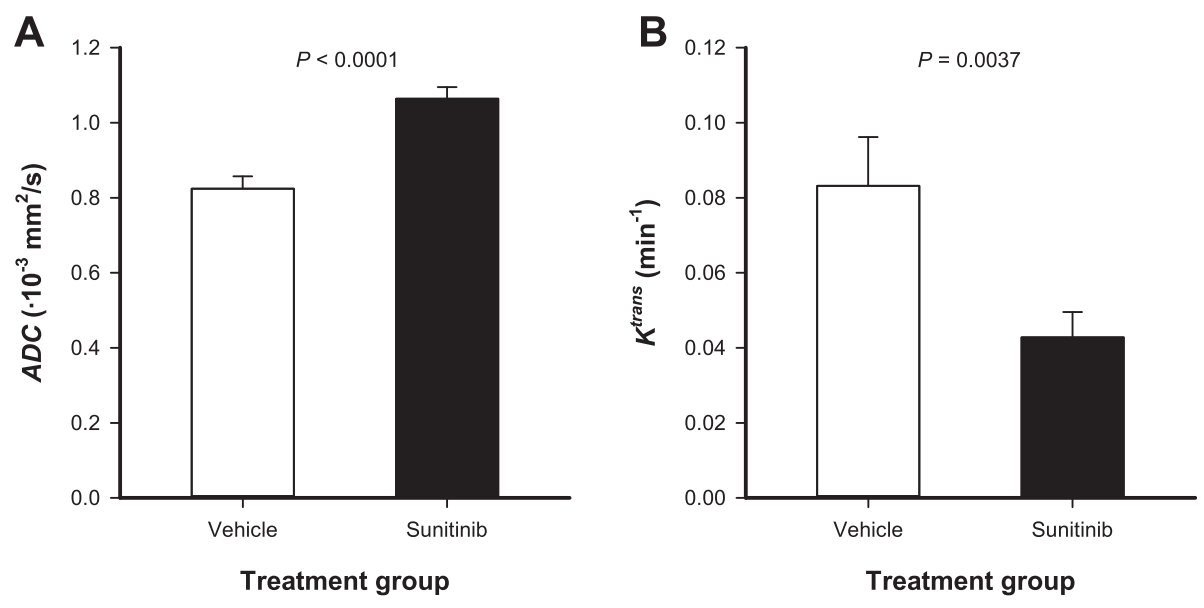

Figure 4 Sunitinib treatment increased $A D C$ and reduced $K^{\text {trans }}$ values. ADC $(\mathbf{A})$ and $K^{\text {trans }}(\mathbf{B})$ in untreated and sunitinib-treated A-07 tumors. Columns, means of 14-15 tumors; bars, SEM.

effects of blood perfusion, as recommended by Padhani et al. [30]. It is therefore unlikely that the ADC values reported here were significantly influenced by vascular effects. The present study thus strongly suggests that $\mathrm{ADC}$ and $K^{\text {trans }}$ reflected different physiological parameters, illustrating that it may be beneficial to combine DW-MRI and DCE-MRI when evaluating effects of antiangiogenic treatment.

It has been suggested that antiangiogenic agents including sunitininib can normalize tumor vasculature and microenvironment and hence sensitize tumors to conventional therapy $[4,31]$. Thus antiangiogenic treatment has been shown to enhance blood perfusion, improve oxygenation, and lower IFP in some tumor models [2,3]. In other tumor models, antiangiogenic agents have failed to normalize the vasculature and have induced hypoxia $[10,11]$. In the current study, sunitinib treatment reduced microvascular density, increased hypoxic fraction, induced necrosis, and did not alter IFP. Consequently, the treatment schedule applied here resulted in changes in the tumor microenvironment that argue against treatmentinduced normalization. This observation is in line with our previous experience with A-07 and R-18 human melanoma xenografts growing in dorsal window chambers [11]. In that study, tumors were treated with two different sunitinib doses and the effect was assessed multiple times during the treatment period. The treatments did not improve vascular function at any time point, suggesting that sunitinib cannot normalize tumor vasculature in these melanoma xenografts.

In tumors where antiangiogenic treatment induces hypoxia, neoadjuvant antiangiogenic therapy is expected to reduce the effect of radiation and chemotherapy $[7,8]$. In contrast, neoadjuvant antiangiogenic therapy has been shown to enhance the effect of radiation or chemotherapy in preclinical tumors where antiangiogenic treatment normalizes the vasculature and the microenvironment $[2,3]$. The current study suggests that DW-MRI and DCEMRI can be used to identify tumors where antiangiogenic treatment does not normalize the microenvironment. These tumors respond to antiangiogenic treatment with reduced $K^{\text {trans }}$ and increased ADC. Interestingly, increased $K^{\text {trans }}$ and reduced ADC have been reported in tumors where antiangiogenic treatment has normalized the vasculature and the microenvironment $[14,32]$.

Vascular normalization is a transient effect because tumors can switch to other angiogenesis pathways and become resistant to antiangiogenic agents. The duration of improved tumor oxygenation is also expected to be limited because the beneficial effects of vascular normalization may be balanced by severe vascular regression after prolonged exposure to antiangiogenic agents [31]. Winkler et al. demonstrated that VEGFR-2 blockade enhanced the effect of radiation when the tumors were irradiated during the time window when the antiangiogenic agent normalized the vasculature and improved oxygenation [3]. They also showed that VEGFR-2 blockade did not enhance the effect of radiation when tumors were irradiated before or after this time window, suggesting that the timing of combination therapies may be crucial to achieve maximal antitumor effect. Previous studies suggest that DW-MRI and DCE-MRI are sensitive to vascular normalization $[14,32]$, and the current study suggests that these techniques are also sensitive to microenvironmental effects that indicate no normalization. Taken together, these studies suggest that DW-MRI and DCE-MRI may be used to monitor the effect of antiangiogenic treatment to identify a potential normalization window.

\section{Conclusions}

Previous studies have suggested that DW-MRI and DCEMRI are sensitive to vascular normalization. The current 
study demonstrates that these techniques also are sensitive to treatment-induced changes in the tumor microenvironment that indicate no normalization, suggesting that these imaging techniques may be used to identify both tumors where antiangiogenic treatment normalizes the microenvironment and tumors where antiangiogenic treatment does not normalize the microenvironment. Furthermore, the current study demonstrates that DWMRI and DCE-MRI are sensitive to treatment-induced changes in the tumor microenvironment that occur before tumor size is affected, suggesting that these techniques can predict tumor response to antiangiogenic treatment before treatment-induced reductions in tumor size can be detected.

\section{Competing interests}

The authors declare that they have no competing interests.

\section{Authors' contributions}

JVG, TH, TGS, and EKR conceived and designed the study. JVG, VP, and TH performed the experiments. JVG, VP, TH, and EKR analyzed and interpreted the data. JVG and EKR wrote the manuscript. All authors read and approved the final manuscript.

\section{Acknowledgements}

Financial support was received from the Norwegian Cancer Society and the South-Eastern Norway Regional Health Authority.

Received: 21 October 2013 Accepted: 14 November 2013

Published: 19 November 2013

\section{References}

1. Jia Y, Liu M, Huang W, Wang Z, He Y, Wu J, Ren S, Ju Y, Geng R, Li Z: Recombinant human endostatin endostar inhibits tumor growth and metastasis in a mouse xenograft model of colon cancer. Pathol Oncol Res 2012, 18:315-323.

2. Dickson PV, Hamner JB, Sims TL, Fraga CH, Ng CY, Rajasekeran S, Hagedorn NL, McCarville MB, Stewart CF, Davidoff AM: Bevacizumab-induced transient remodeling of the vasculature in neuroblastoma xenografts results in improved delivery and efficacy of systemically administered chemotherapy. Clin Cancer Res 2007, 13:3942-3950.

3. Winkler F, Kozin SV, Tong RT, Chae SS, Booth MF, Garkavtsev I, Xu L, Hicklin DJ, Fukumura D, di Tomaso E, et al: Kinetics of vascular normalization by VEGFR2 blockade governs brain tumor response to radiation: role of oxygenation, angiopoietin-1, and matrix metalloproteinases. Cancer Cell 2004, 6:553-563.

4. Czabanka M, Vinci M, Heppner F, Ullrich A, Vajkoczy P: Effects of sunitinib on tumor hemodynamics and delivery of chemotherapy. Int I Cancer 2009, 124:1293-1300.

5. Morgan B, Horsfield MA, Steward WP: The role of imaging in the clinical development of antiangiogenic agents. Hematol Oncol Clin North Am 2004, 18:1183-1206.

6. Li SP, Padhani AR: Tumor response assessments with diffusion and perfusion MRI. J Magn Reson Imaging 2012, 35:745-763.

7. Horsman MR, Siemann DW: Pathophysiologic effects of vascular-targeting agents and the implications for combination with conventional therapies. Cancer Res 2006, 66:11520-11539.

8. Brown JM, Giaccia AJ: The unique physiology of solid tumors: opportunities (and problems) for cancer therapy. Cancer Res 1998, 58:1408-1416

9. Heldin $\mathrm{CH}$, Rubin $\mathrm{K}$, Pietras K, Östman A: High interstitial fluid pressure an obstacle in cancer therapy. Nat Rev Cancer 2004, 4:806-813.

10. Franco M, Man S, Chen L, Emmenegger U, Shaked Y, Cheung AM, Brown AS, Hicklin DJ, Foster FS, Kerbel RS: Targeted anti-vascular endothelial growth factor receptor-2 therapy leads to short-term and long-term impairment of vascular function and increase in tumor hypoxia. Cancer Res 2006, 66:3639-3648.

11. Gaustad JV, Simonsen TG, Leinaas MN, Rofstad EK: Sunitinib treatment does not improve blood supply but induces hypoxia in human melanoma xenografts. BMC Cancer 2012, 12:388.

12. Yankeelov TE, Arlinghaus LR, Li X, Gore JC: The role of magnetic resonance imaging biomarkers in clinical trials of treatment response in cancer. Semin Oncol 2011, 38:16-25.

13. Padhani AR: Diffusion magnetic resonance imaging in cancer patient management. Semin Radiat Oncol 2011, 21:119-140.

14. Batchelor TT, Duda DG, di Tomaso E, Ancukiewicz M, Plotkin SR, Gerstner E, Eichler AF, Drappatz J, Hochberg FH, Benner T, et al: Phase II study of cediranib, an oral pan-vascular endothelial growth factor receptor tyrosine kinase inhibitor, in patients with recurrent glioblastoma. J Clin Oncol 2010, 28:2817-2823.

15. Loveless ME, Lawson D, Collins M, Nadella MV, Reimer C, Huszar D, Halliday J, Waterton JC, Gore JC, Yankeelov TE: Comparisons of the efficacy of a Jak1/2 inhibitor (AZD1480) with a VEGF signaling inhibitor (cediranib) and sham treatments in mouse tumors using DCE-MRI, DW-MRI, and histology. Neoplasia 2012, 14:54-64.

16. Tofts PS, Brix G, Buckley DL, Evelhoch JL, Henderson E, Knopp MV Larsson HB, Lee TY, Mayr NA, Parker GJ, et al: Estimating kinetic parameters from dynamic contrast-enhanced T(1)-weighted MRI of a diffusable tracer: standardized quantities and symbols. J Magn Reson Imaging 1999, 10:223-232.

17. Tofts PS: Modeling tracer kinetics in dynamic Gd-DTPA MR imaging J Magn Reson Imaging 1997, 7:91-101.

18. Egeland TA, Simonsen TG, Gaustad JV, Gulliksrud K, Ellingsen C, Rofstad EK: Dynamic contrast-enhanced magnetic resonance imaging of tumors: preclinical validation of parametric images. Radiat Res 2009, 172:339-347.

19. Roskoski R Jr: Sunitinib: a VEGF and PDGF receptor protein kinase and angiogenesis inhibitor. Biochem Biophys Res Commun 2007, 356:323-328.

20. Demetri GD, van Oosterom AT, Garrett CR, Blackstein ME, Shah MH, Verweij J, McArthur G, Judson IR, Heinrich MC, Morgan JA, et al: Efficacy and safety of sunitinib in patients with advanced gastrointestinal stromal tumour after failure of imatinib: a randomised controlled trial. Lancet 2006, 368:1329-1338.

21. Motzer RJ, Hutson TE, Tomczak P, Michaelson MD, Bukowski RM, Oudard S, Negrier S, Szczylik C, Pili R, Bjarnason GA, et al: Overall survival and updated results for sunitinib compared with interferon alfa in patients with metastatic renal cell carcinoma. J Clin Oncol 2009, 27:3584-3590.

22. Raymond E, Hammel P, Dreyer C, Maatescu C, Hentic O, Ruszniewski P, Faivre S: Sunitinib in pancreatic neuroendocrine tumors. Target Oncol 2012, 7:117-125.

23. Rofstad EK: Orthotopic human melanoma xenograft model systems for studies of tumour angiogenesis, pathophysiology, treatment sensitivity and metastatic pattern. Br J Cancer 1994, 70:804-812.

24. Benjaminsen IC, Graff BA, Brurberg KG, Rofstad EK: Assessment of tumor blood perfusion by high-resolution dynamic contrast-enhanced MRl: a preclinical study of human melanoma xenografts. Magn Reson Med 2004, 52:269-276.

25. Hittmair K, Gomiscek G, Langenberger K, Recht M, Imhof H, Kramer J: Method for the quantitative assessment of contrast agent uptake in dynamic contrast-enhanced MRI. Magn Reson Med 1994, 31:567-571.

26. Ozerdem U, Hargens AR: A simple method for measuring interstitial fluid pressure in cancer tissues. Microvasc Res 2005, 70:116-120.

27. Rofstad EK, Måseide K: Radiobiological and immunohistochemical assessment of hypoxia in human melanoma xenografts: acute and chronic hypoxia in individual tumours. Int J Radiat Biol 1999, 75:1377-1393.

28. Gaustad JV, Brurberg KG, Simonsen TG, Mollatt CS, Rofstad EK: Tumor vascularity assessed by magnetic resonance imaging and intravital microscopy imaging. Neoplasia 2008, 10:354-362.

29. Senger DR, Van De WL, Brown LF, Nagy JA, Yeo KT, Yeo TK, Berse B, Jackman RW, Dvorak AM, Dvorak HF: Vascular permeability factor (VPF, VEGF) in tumor biology. Cancer Metastasis Rev 1993, 12:303-324.

30. Padhani AR, Liu G, Koh DM, Chenevert TL, Thoeny HC, Takahara T, Dzik-Jurasz A, Ross BD, Van CM, Collins D, et al: Diffusion-weighted magnetic resonance imaging as a cancer biomarker: consensus and recommendations. Neoplasia 2009, 11:102-125. 
31. Jain RK: Normalization of tumor vasculature: an emerging concept in antiangiogenic therapy. Science 2005, 307:58-62.

32. Sorensen AG, Emblem KE, Polaskova P, Jennings D, Kim H, Ancukiewicz M, Wang M, Wen PY, Ivy P, Batchelor TT, et al: Increased survival of glioblastoma patients who respond to antiangiogenic therapy with elevated blood perfusion. Cancer Res 2012, 72:402-407.

doi:10.1186/1756-9966-32-93

Cite this article as: Gaustad et al.: Magnetic resonance imaging identifies early effects of sunitinib treatment in human melanoma xenografts.

Journal of Experimental \& Clinical Cancer Research 2013 32:93.

\section{Submit your next manuscript to BioMed Central and take full advantage of:}

- Convenient online submission

- Thorough peer review

- No space constraints or color figure charges

- Immediate publication on acceptance

- Inclusion in PubMed, CAS, Scopus and Google Scholar

- Research which is freely available for redistribution 